\title{
Abuso de Drogas: Generalidades Neurobiológicas y Terapéuticas
}

\section{Drug abuse: neurobiological and terapeutic generalities}

\author{
María Carolina Velásquez-Martínez ${ }^{1}$ \\ Universidad Industrial de Santander, Colombia
}

\author{
José G. Ortiz ${ }^{2}$ \\ Universidad de Puerto Rico, Puerto Rico
}

Resumen. El abuso de drogas es una enfermedad caracterizada por la urgencia de obtener y consumir una sustancia determinada independientemente de sus consecuencias negativas. Existen factores biológicos, sociales y psicológicos que predisponen a un individuo al abuso de drogas los adolescentes son los más vulnerables porque su cerebro no ha madurado completamente y están bajo presiones tanto sociales como psicológicas. Es por esto último que la prevención es aún más efectiva a esta edad. Las drogas de abuso alteran principalmente el sistema mesocorticolimbico; la dopamina y el glutamato desempeñan un papel importante en este circuito que normalmente median comportamientos de motivación. Se cree que las sustancias de abuso modifican este circuito usando mecanismos similares al aprendizaje normal resultando en aprendizaje disfuncional. Dichos cambios son a largo plazo y pueden ser evocados por eventos que recuerden la droga, en la ausencia de la misma. Un manejo multidisciplinario es necesario ya que el abuso de drogas tiene componentes biológicos, sociales y psicológicos. Las terapias cognitivas y comportamentales son comúnmente complementadas con agentes farmacológicos con los que se manejan los síntomas específicos de retirada y abstinencia.

Palabras clave. Adicción, sistema mesocorticolimbico, dopamina, generalidades terapéuticas.

Abstract. Drug abuse is an illness characterized by the urge to obtain and consume a given substance regardless of the negative consequences. There are biological, social and psychological factors that predispose an individual to abuse drugs. Adolescents are most vulnerable because their brain has not fully matured yet and there are strong social and psychological pressures. Thus, prevention is most effective at this age. Most abused drugs target the brain's mesocorticolimbic system. Dopamine and glutamate play a major role in this circuit that normally mediates motivated behaviors. It is thought that adicctive substances modify this circuit using mechanisms similar to normal learning resulting in dysfunctional learning. Such changes are very long lasting and can be evoked by events reminiscent of the drug abuse, in the absence of the drug. A multidisciplinary approach is needed because drug abuse has biological, social and psychological components. Cognitive and behavioral therapies are often paired with pharmacological agents that address specific drug withdrawal and abstinence symptoms.

Keywords. Addiction, mesocorticolimbic system, dopamine, therapeutic generalities.

\footnotetext{
${ }^{1}$ María Carolina Velásquez-Martínez, Laboratorio de Neurociencias y Comportamiento, Departamento de Ciencias Básicas, Escuela de Medicina, Facultad de Salud, Universidad Industrial de Santander. Bucaramanga, Colombia. Dirección Postal: Calle 9 \# 27, Bucaramanga, Santander 680002, Colombia Email: carolinav24@yahoo.es

${ }^{2}$ José G. Ortiz, Departamento de Farmacología y Toxicología, Escuela de Medicina, Universidad de Puerto Rico. E-mail: jg_ortiz@yahoo.com
}

\section{()ㅇ $\Theta \Theta$}

Esta obra está bajo una Licencia Creative Commons Atribución-NoComercial-SinDerivar 4.0 Internacional. 


\section{Introducción}

El presente artículo pretende presentar una visión general del complejo tópico que es la adicción y abuso de drogas. En este escrito, "abuso de sustancias" se refiere a la búsqueda compulsiva y al consumo de una sustancia independientemente de las consecuencias que pueda tener en la funcionalidad del individuo (NIDA, 2008)

Con frecuencia se utilizan los términos "fármacodependiente" y "adicción a drogas" como equivalentes, la "fármacodependencia" se refiere a un síndrome de retirada cuando el uso del fármaco es descontinuado abruptamente. Sin embargo, esta "equivalencia" lleva a errores; a saber, hay sustancias que se abusan sin que haya dependencia (e.g. LSD) y hay fármacodependencias totalmente funcionales y hasta necesarias (e.g. insulina en el manejo de diabetes).

Otra fuente de confusión, es la legalidad de la sustancia que es abusada, la legalidad de una sustancia no modifica sus propiedades farmacológicas ni impiden el que sean abusadas, siendo el alcohol, el mejor ejemplo.

\section{Características}

Etapas. En general, el proceso de la generación de la adicción a sustancias tiene varias etapas: experimentación/uso ocasional, aumento en consumo y el abuso; además factores sociales y familiares como condiciones en el hogar, en la escuela o en el vecindario, pueden influenciar el comienzo de uso y abuso de drogas. Todos estos factores, incluyendo los factores biológicos, deben ser abordados de forma individualizada para aumentar la probabilidad de tener un tratamiento eficaz de la drogadicción.

El proceso adicción a una droga puede darse a cualquier edad pero entre más temprano se comience el consumo, mayor será probabilidad de generar un abuso mayor dependencia de la droga, ya que el desarrollo del sistema nervioso central aún no concluye hasta entrada la segunda década de vida (Gogtay et al., 2004), de esta manera, la adolescencia es el punto más frágil para generar el abuso de sustancias y su adicción. Adicionalmente a esta edad, la influencia del hogar, la familia y los compañeros de escuela pueden generar un ambiente socialmente negativo, precipitando el consumo y el posterior abuso de drogas. Se ha observado que la prevención e intervención temprana sobre el consumo de drogas en la adolescencia ha sido una estrategia que puede evitar mayores consecuencias (NIDA, 2004).

Neurobiología. Uno de los sustratos anatómicos más importantes asociados con comportamientos de adicción es el sistema mesocorticolímbico, este sistema está compuesto por neuronas dopaminérgicas (DA) que proyectan desde el área ventrotegmental (VTA) hacia la corteza prefrontal (PFC) y el núcleo accumbens (NAcc) (Dahlstrom and Fuxe, 1964; Ungerstedt, 1971). La activación de las neuronas DA del VTA está implicada en el comportamiento de motivación y en acciones de refuerzo de las drogas de abuso (Schultz, 2002; Kauer, 2004; Grace, Floresco, Goto \& Lodge 2007). La pérdida de control del uso de la droga es asociada principalmente a la disminución de la función por parte de la PFC; mientras que la búsqueda de la sustancia de abuso es asociada con el NAcc. Diferentes estructuras cerebrales como la amígdala, hipocampo e hipotálamo, que se encuentran asociadas con aprendizaje, memoria y comportamientos emocionales, contribuyen en el desarrollo de la dependencia y adicción (Everitt \& Robbins, 2005; Haile, De la Garza \& Mahoney, 2012).

En este circuito la DA y el glutamato juegan un papel protagónico. Dentro del VTA predominan neuronas DA que están bajo la influencia de aferencias glutamatergicas provenientes de PFC y otras estructuras (Baik, 2013). La acción del glutamato en esta área contribuye en cambios cognitivos, estrés y recompensa; y al mismo tiempo es crítico en los efectos que inducen las drogas de abuso (Ungless, Whistler, Malenka \& Bonci, 2001; You,Wang, Zitzman, Azari \& Wise, 2007; Wise, 2009).

La literatura ha demostrado que la excitación generada por glutamato puede modificar la actividad eléctrica de las neuronas DA del VTA (Murase, Grenhoff, Chouvet, Gonon, \& Svensson, 1993; Georges \& Aston-Jones, 2002; Lodge \& Grace, 2006). Estos hallazgos dejan ver que el control excitatorio sobre las células DA es de gran importancia fisiológica y patológica.

Se han descrito cambios moleculares que acompañan las distintas etapas de la adicción en modelos animales y en 
humanos. Se ha demostrado también que el uso continuo y el abuso de drogas lleva a cambios en la neurotransmisión de DA en el cerebro (Letchworth, Nader, Smith, Friedman \& Porrino, 2001; Volkow et al., 2001; Gould, Porrino \& Nader, 2012). De estos estudios en modelos animales y humanos surgen varios hallazgos importantes: 1 . todas las drogas que son abusadas modifican el circuito VTA-Accumbens-PFC; 2. la integridad de ese circuito es imprescindible para que se manifieste la adicción; 3. la adicción está acompañada por unos cambios muy duraderos una vez que ésta se establece (Goldstein \& Volkow, 2002; Wise, 2002; Everitt \& Robbins, 2005; Hyman, 2005). Tan impactantes son estos cambios que pueden re-activar la estimulación de dicho circuito sólo con la imagen del consumo.

El entendimiento actual apunta no solamente a que las drogas de abuso se apoderan de este circuito causando cambios duraderos, sino que el desarrollo de la adicción es muyparecido a un aprendizaje disfuncional. Como resultado, la asociación entre la droga y los elementos ambientales que tienen relación con el consumo de la droga puede disparar el deseo de consumir y de comportamientos compulsivos de búsqueda. Estos comportamientos llevan a la recaída al consumo y a pérdida del control voluntario de evitación de la droga; dichas modificaciones en el comportamiento, que según se ha observado, son duraderas, son reflejo de cambios de respuestas neuronales en el circuito de recompensa, lo que sugiere que el comportamiento de consumo de la droga se convierte en un refuerzo de este aprendizaje disfuncional (Wolf, 2002).

\section{Estrategias terapénticas}

Es imprescindible que el manejo de las adicciones consista en un enfoque multidisciplinario a raíz de que ésta tiene componentes biológicos, psicológicos y sociales. Las estrategias que se pueden abordar van acompañadas tanto de medicamentos que ayudan a tratar el síndrome de abstinencia y evitar recaídas.

El manejo farmacológico está centralizado en disminuir los síntomas de depresión, ansiedad, inquietud y dificultad para dormir (NIDA, 2008; Lefio, Villarroel, Rebolledo, Zamorano \& Rivas, 2013). Por otro lado, el tratamiento medicado aumenta su eficacia con la ayuda de terapias conductuales.
El manejo farmacológico de la adicción puede organizarse en tres etapas: 1. manejo de la intoxicación, 2. manejo de la retirada y 3. mantenimiento de la abstinencia (NIDA, 2010).

Por otra parte, el manejo de la intoxicación está enfocado en mantener las funciones vitales del individuo. En el caso de opiáceos (ej heroína), el uso de antagonistas como naltrexona o naloxona ayudan a revertir los efectos de esas drogas (Habal, 2013; Stephens, 2014). De igual manera, el flumazenil es útil en revertir la toxicidad de benzodiacepinas (Scher, 2014).

Por otra parte, el manejo farmacológico de la retirada está basado en aliviar los síntomas de ésta y puede acompañarse de drogas cuyo efecto sea prolongado pero su potencia menor. El uso de la metadona y la buprenorfina son ejemplos para la retirada de los opiáceos; en el caso de nicotina, los parchos son una posible alternativa.

Es importante señalar que mantener al usuario libre de consumo de droga es una tarea difícil en la que el enfoque farmacológico sólo no es suficiente.

Dentro de las estrategias terapéuticas conductuales se encuentran la realización de terapias cognitivoconductual, entrevistas de motivación y terapias grupales (NIDA, 2008, 2010; Engle, 2011; Smedslund et al., 2011).

La terapia cognitivo-conductual tiene como objetivos identificar y modificar las actitudes y comportamientos relacionados al consumo y abuso de drogas (Carroll et al., 2006). Para poder manejar las recaídas, se requiere adquirir destrezas que le hagan contrapeso al aprendizaje disfuncional causado por las drogas. La literatura muestra que la ejecución de terapias cognitivo-conductuales como tratamiento en abuso de sustancias presentan disminución de adherencia y no tienen el efecto esperado a largo plazo (Magill \& Ray, 2009; Lefio et al., 2013). Sin embargo el resultado positivo de esta terapia, como la disminución de recaídas, aumenta en la medida que se combine con tratamiento psicosocial adicional (Magill \& Ray, 2009).

La terapia de motivación es una terapia psicológica, que dirigida por el consejero y centrada en el sujeto, con el fin de aumentar la motivación intrínseca hacia el cambio 
mediante la exploración y resolución de comportamientos y pensamientos ambivalentes (Apodaca \& Longabaugh, 2009). Durante las sesiones de terapia, el consejero interviene para ayudar al sujeto a identificar y comprender el beneficio de dejar las drogas y las consecuencias si no lo hace.

El consejero tiene un rol activo guiando al sujeto en objetivos a corto y largo plazo y evaluando constantemente su acción sobre estos (Engle, 2011; Smedslund et al., 2011). Adicionalmente, el consejero debe desarrollar empatía con el sujeto para comprender su punto de vista y llevarlo a que perciba la discrepancia entre lo que se es y lo que se quiere ser. Cabe destacar que en este tipo de terapia de motivación el sujeto debe mostrar disposición a la salida de su problema de abuso y adicción de sustancias (Apodaca \& Longabaugh, 2009; Smedslund et al., 2011).

Las terapias grupales, adaptadas para ejecutarse en conjunto con terapias cognitivo-conductuales y motivacionales, son tratamientos que ayudan a reforzar comportamientos sociales que pueden ayudar a mantener al individuo un estilo de vida sin drogas y fomenta la abstinencia (NIDA, 2010). Sin embargo, el manejo de estos grupos terapéuticos por parte del terapeuta debe ser cuidadoso y monitoreado para evitar desmotivación y deserción amplia del programa.

Por lo anteriormente mencionado es importante mantener un abordaje multidisciplinario para el tratamiento de la adicción a drogas o sustancias, complementado con estrategias farmacológicas y psicológicas para su mayor efectividad, revisando periódicamente la adherencia de los sujetos al tratamiento para así garantizar el éxito de éste.

\section{Referencias}

Apodaca, TR. \& Longabaugh, R. (2009) Mechanisms of change in motivational interviewing: a review and preliminary evaluation of the evidence. Addiction, 104, 705-715.

Baik, JH. (2013) Dopamine signaling in reward-related behaviors. Front Neural Circuits, 7, 152.

Carroll, KM., Easton, CJ., Nich, C., Hunkele, KA., Neavins, TM., Sinha, R., Ford, HL., Vitolo, SA., Doebrick, CA. \& Rounsaville, BJ. (2006). The use of contingency management and motivational/skills- building therapy to treat young adults with marijuana dependence. J Consult Clin Psychol, 74, 955-966.

Dahlstrom, A. \& Fuxe, K. (1964). Localization of monoamines in the lower brain stem. Experientia, 20,398-399.

Engle, B. (2011) Review: motivational interviewing reduces substance use compared with no treatment in substancedependent individuals. Evid Based Ment Health, 14, 116.

Everitt, BJ. \& Robbins, TW. (2005). Neural systems of reinforcement for drug addiction: from actions to habits to compulsion. Nat Neurosci, 8,1481-1489.

Georges, F. \& Aston-Jones, G. (2002). Activation of ventral tegmental area cells by the bed nucleus of the stria terminalis: a novel excitatory amino acid input to midbrain dopamine neurons. J Neurosci, 22, 5173-5187.

Gogtay, N., Giedd, JN., Lusk, L., Hayashi, KM., Greenstein, D., Vaituzis, AC., Nugent TF., Herman, DH., Clasen, LS., Toga, AW., Rapoport, JL., \& Thompson, PM. (2004). Dynamic mapping of human cortical development during childhood through early adulthood. Proc Natl Acad Sci USA 101, 8174-8179.

Goldstein, RZ. \& Volkow, ND. (2002). Drug addiction and its underlying neurobiological basis: neuroimaging evidence for the involvement of the frontal cortex. Am J Psychiatry, 159, 1642-1652.

Gould RW., Porrino LJ., \& Nader MA. (2012). Nonhuman Primate Models of Addiction and PET Imaging: Dopamine System Dysregulation. Curr Top Behav Neurosci, 11, 25-44.

Grace, AA., Floresco, SB., Goto, Y. \& Lodge, DJ. (2007). Regulation of firing of dopaminergic neurons and control of goal-directed behaviors. Trends Neurosci, 30, 220-227.

Habal, R. (2013). Heroin Toxicity. Retrieved from http:// emedicine.medscape.com/article/166464-overview

Haile, CN., Mahoney, JJ., Newton, TF. \& De La Garza, R., (2012). Pharmacotherapeutics directed at deficiencies associated with cocaine dependence: focus on dopamine, norepinephrine and glutamate. Pharmacol Ther, 134, 260-277. 
Hyman, SE. (2005). Addiction: a disease of learning and memory. Am J Psychiatry, 162, 1414-1422.

Kauer, JA. (2004). Learning mechanisms in addiction: synaptic plasticity in the ventral tegmental area as a result of exposure to drugs of abuse. Annu Rev Physiol, 66, 447-475.

Lefio, LA., Villarroel, SR., Rebolledo, C., Zamorano P. \& Rivas, K. (2013). Effective interventions in the problematic use of alcohol and other drugs. Rev Panam Salud Publ, 34, 257-266.

Letchworth, SR., Nader, MA., Smith, HR., Friedman, DP. \& Porrino, LJ. (2001). Progression of changes in dopamine transporter binding site density as a result of cocaine self-administration in thesus monkeys. $J$ Neurosci, 21, 2799-2807.

Lodge, DJ. \& Grace, AA. (2006). The laterodorsal tegmentum is essential for burst firing of ventral tegmental area dopamine neurons. Proc Natl Acad Sci USA, 103, 5167-5172.

Magill, M. \& Ray, LA. (2009). Cognitive-behavioral treatment with adult alcohol and illicit drug users: a meta-analysis of randomized controlled trials. J Stud Alcohol Drugs, 70, 516-527.

Murase, S., Grenhoff J., Chouvet, G., Gonon, FG. \& Svensson, T. (1993). Prefrontal cortex regulates burst firing and transmitter release in rat mesolimbic dopamine neurons studied in vivo. Neurosci Lett, 157, 53-56.

NIDA (2004). Cómo prevenir el uso de drogas en los niños y los adolescentes. Retrieved from http://www.drugabuse.gov/ es/publicaciones/como-prevenir-el-uso-de-drogas.

NIDA (2008). Las drogas, el cerebro y el comportamiento: la ciencia de la adicción. National Institute on Drug Abuse. Retrieved from http://www.drugabuse. gov/es/publicaciones/las-drogas-el-cerebro-y-elcomportamiento

NIDA (2010). Principios de tratamientos para la drogadicción: Una guia basada en las investigaciones. Retrieved from http://www.drugabuse.gov/es/publicaciones/ principios-de-tratamientos-para-la-drogadiccion
Scher, LM. (2014). Sedative, Hypnotic, Anxiolytic Use Disorders. Retrieved from http://emedicine. medscape.com/article/290585-overview\#showall

Schultz, W. (2002). Getting formal with dopamine and reward. Neuron, 36, 241-263.

Smedslund, G., Berg RC., Hammerstrom, KT., Steiro, A., Leiknes, KA., Dahl, HM., Karlsen, K. (2011). Motivational interviewing for substance abuse. Cochrane Database Syst Rev CD008063. doi: 10.1002/14651858.CD008063.pub2.

Stephens, E. (2014). Opioid Toxicity. In: Medscape Reference Drugs and Diseases. Retrieved from http://emedicine. medscape.com/article/815784-overview\#showall

Ungerstedt, U. (1971). Stereotaxic mapping of the monoamine pathways in the rat brain. Acta Physiol Scand Suppl, 367, 1-48.

Ungless, MA., Whistler, JL., Malenka, RC. \& Bonci, A. (2001). Single cocaine exposure in vivo induces long-term potentiation in dopamine neurons. Nature, 411, 583-587.

Volkow, ND., Chang, L., Wang, GJ., Fowler, JS., Franceschi, D., Sedler, M., Gatley, SJ., Miller, E., Hitzemann, R., Ding, YS. \& Logan, J. (2001). Loss of dopamine transporters in methamphetamine abusers recovers with protracted abstinence. $J$ Neurosci, 21, 9414-9418.

Wise, RA. (2002). Brain reward circuitry: insights from unsensed incentives. Neuron, 36, 229-240.

Wise, RA. (2009). Ventral tegmental glutamate: a role in stress-, cue-, and cocaine-induced reinstatement of cocaine-seeking. Neuropharmacology 56 Suppl 1, 174-176.

Wolf, ME. (2002). Addiction: making the connection between behavioral changes and neuronal plasticity in specific pathways. Mol Interv 2, 146-157.

You, ZB., Wang, B., Zitzman, D., Azari, S., \& Wise, RA. (2007). A role for conditioned ventral tegmental glutamate release in cocaine seeking. J Neurosci 27, 10546-10555.

Recibido: 04 de marzo, 2014 Aceptado: 20 de agosto, 2014 